Review

\title{
The Etiological Role of Microglia in Autism Spectrum Disorder: A Possible Route for Early Intervention
}

\author{
Ryan Slovak and Nitya G. Chakraborty \\ Department of Medicine, University of Connecticut School of Medicine, \\ 263 Farmington Avenue, Farmington, CT 06032, USA
}

Article history

Received: 14-06-2017

Revised: 19-06-2017

Accepted: 23-06-2017

Corresponding Author: Nitya G. Chakraborty Department of Medicine, University of Connecticut School of Medicine, 263

Farmington Avenue,

Farmington, CT 06032, USA

Tell: (860) 679-1446

Email: chakraborty@uchc.edu

\begin{abstract}
Autism Spectrum Disorder (ASD) is a dreaded diagnosis. The treatment options are sparse and our knowledge of its etiology is woefully lacking. The purpose of this review is to outline a unifying pathway for the pathogenesis of Autism Spectrum Disorder and to describe how this sequence could be exploited to offer early intervention to patients at high risk of developing ASD. Specifically, we will describe how gestational insults can alter the lifelong functioning of fetal microglia. Those aberrant microglia are unable to properly fulfill their roles in cortical differentiation as well as synaptogenesis and the resulting cortical disorganization plus dendritic overgrowth may be responsible for the characteristic Autistic phenotype. This pathogenic pathway presents several opportunities for intervention. If applied early enough, it is possible that these therapies could alleviate some of the symptoms of ASD.
\end{abstract}

Keywords: Autism, Microglia, Gestational Insult, Intervention

\section{Introduction}

Autism Spectrum Disorder (ASD) encompasses a broad range of heterogeneous neuro developmental disabilities. The newly defined spectrum includes Autism as well as Asperger's and Rett syndrome (Harris, 2016). In addition to sharing various degrees of archetypal social impairment, these disorders are characterized by early neural overgrowth and dysfunction (Feldman et al., 2016; Hutsler and Zhang, 2010; Stoner et al., 2014). This pattern of excessive growth has been shown to reverse and recede by adulthood (Courchesne et al., 2011; 2001). Though research has implicated both genetics and environmental stressors in the pathogenesis of ASD, an overarching etiology remains elusive (Harris, 2016; Stoner et al., 2014). What is clear is that although the diagnosis of ASD is not usually made until the child is around 3 years old, the developmental derangement likely begins in the womb (Gardener et al., 2009; Stoner et al., 2014). As proof of this, distinct "patches" of cortical disorganization have been identified in the brains of ASD patients (Stoner et al., 2014). This indicates that the formation and differentiation of cortical layers that normally occurs in the prenatal period is somehow deranged. This new data supports earlier research that indicated that the neuroanatomic abnormalities seen in ASD likely originated during prenatal development
(Bauman and Kemper, 2005). The brain begins developing in the 3rd week of gestation and cortical organization continues throughout the prenatal period (Stiles and Jernigan, 2010). During this time, the developing brain is uniquely susceptible to insults. Alterations that occur in this step of the developmental process will impact the lifelong structure and function of the brain (Connors et al., 2008). Critically, although the basic structure of the brain is laid out in the womb, neurodevelopment continues into adolescence. In particular, the volume of the brain rapidly expands during early childhood and dendritic density peaks between 12 and 36 months of age, before being pruned back by microglial cells (Hutsler and Zhang, 2010; Paolicelli et al., 2011; Wake et al., 2009). In addition to exhibiting patches of cortical disorganization, ASD patients have also been found to have greatly increased brain volume and dendritic spine densities (Hutsler and Zhang, 2010). Therefore, the mechanism responsible for ASD must involve both prenatal cortical organization and postnatal dendritic density regulation.

Microglia take part in both of these functions and are abnormally active in ASD patients (Cherry et al., 2014; Gupta et al., 2014; Morgan et al., 2010). During development, microglia associate with the progenitor zones of the brain where they modulate the assembly of axonal tracts and cortical networks (Squarzoni et al., 2015). 


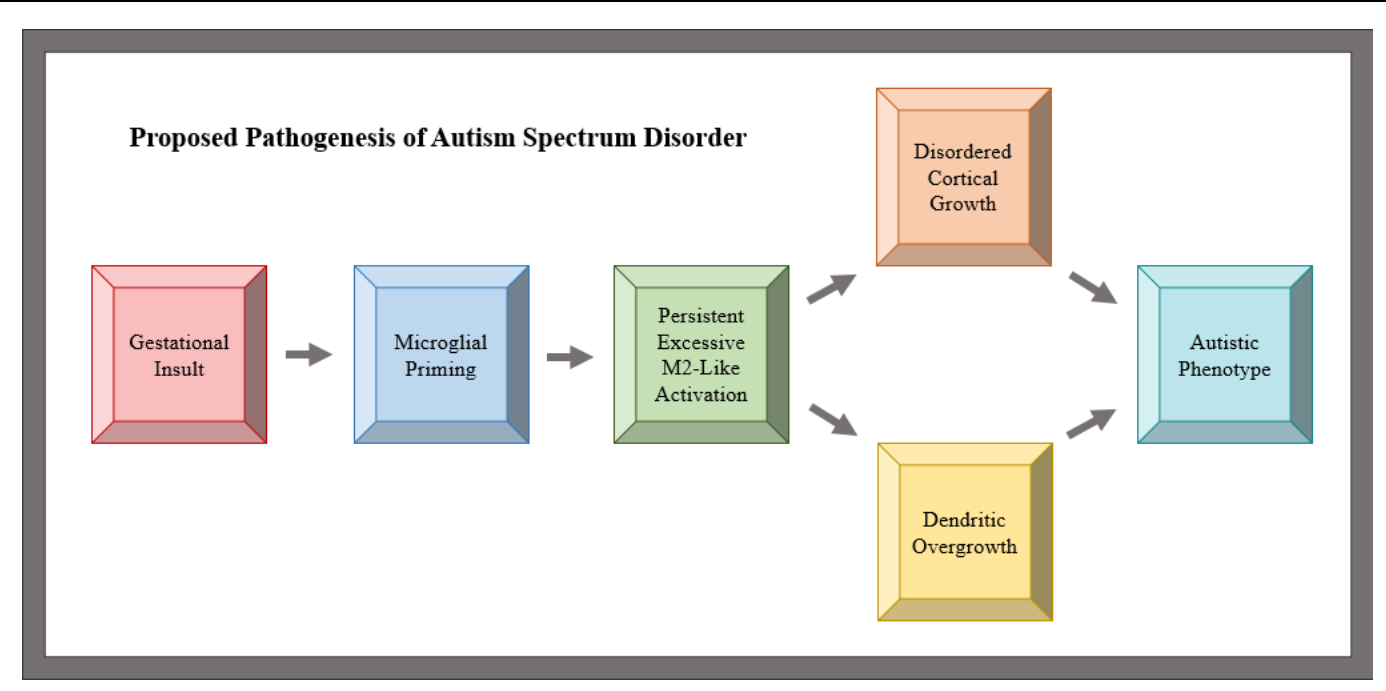

Fig. 1. Flow chart depicting our proposed pathway for the pathogenesis of Autism Spectrum Disorder

Additionally, during the postnatal period, resting state microglia in contact with neuronal synapses are responsible for the pruning of extraneous dendrites (Paolicelli et al., 2011). Moreover, microglia and their associated functions are susceptible to epigenetic alteration induced by gestational insults (Kaminska et al., 2016; Nardone and Elliott, 2016; Squarzoni et al., 2015). We suggest that the pathogenesis of ASD begins with an early gestational insult that disrupts the functioning of fetal microglia via an epigenetic mechanism. These aberrant microglia are then unable to perform their normal roles in cortical organization and synaptic pruning. The result is a malformed and disorganized brain that culminates in the classic symptoms of ASD (Fig. 1).

It is critical that we develop a thorough understanding of ASD. The most recent estimates place the prevalence at 1 in 68 children and it is even higher in boys at 1 in 42 (Christensen et al., 2016). ASD takes a heavy toll on the people it affects, their families and the society that supports them. By the year 2025, it is projected that the annual financial burden of ASD will rise to 461 billion dollars (Leigh and Du, 2015). Therapeutic options are currently limited and relatively ineffective. Educational and behavioral therapies remain the mainstay of treatment, but these options usually leave the defining social dysfunctions intact (Park et al., 2016). Recently the endogenous hormone oxytocin has shown promise as a way to alleviate the social symptoms of ASD, but much work still needs to be done to demonstrate its clinical value (Lee et al., 2015).

\section{Gestational Insults}

Gestational illness has long been associated with neurological changes in the fetus. Brain development begins at 3 weeks, oftentimes before the mother even knows that she is pregnant (Stiles and Jernigan, 2010). This point is particularly salient because $49 \%$ of pregnancies in the United States are unplanned (Finer and Zolna, 2011). Without proper planning, it is possible that these women are exposing themselves to gestational insults without being aware of the harm that they are doing (de La Rochebrochard and Joshi, 2013).

Gestation is a particularly sensitive time for both the mother and the fetus. Not only is the mother entirely responsible for her baby's nutrition, but any stress that the mother experiences may affect the fetus as well. These so-called gestational insults may be chemical, biological, metabolic, physical, or even psychological. Whatever their form, these events are responsible for a multitude of congenital diseases. When it comes to ASD, we are proposing that the precipitating factor must somehow alter the fetal immune system. Maternal stressors likely induce this kind of trans-placental derangement via maladaptive epigenetic changes in the fetus (Ma et al., 2014). When the fetus is exposed to nutritional deficiencies, chemical insults, or stress hormones its body adapts to the new conditions. The epigenetic alterations induced by these events may have long lasting consequences, some of which could result in the neurobehavioral changes seen in ASD.

Maternal psychological stress in particular has a wellestablished connection to neurobehavioral disorders (Talge et al., 2007; Van den Bergh et al., 2005). A specific link to Autism has even been made with higher levels of family discord as well as with stressful life events (Beversdorf et al., 2005; Ward, 1990). Although it remains outside the scope of this review, a possible mechanism for this gestational transfer of stress is via 
maternal cortisol that crosses the placenta and alters the fetal hypothalamic-pituitary-adrenal axis (Matthews, 2002). Technically, psychological stress might be a form of chemical insult, but the specific chemicals have yet to be completely elucidated. Regardless, it has become increasingly apparent that stress and negative emotions can impact the functioning of the immune system (Brod et al., 2014). Maternal stress is a particularly dangerous insult because it is often not under the mother's control.

There have been major concerns from the public about the safety of vaccines and their possible connection to ASD. This concern likely stems from the Wakefield paper originally published in 1998 (Wakefield et al., 1998). This paper was retracted and there has since been no evidence found of a causal link between ASD and vaccinations (Taylor et al., 2014). The only connection between vaccination and ASD that remains is temporal coincidence. Pediatric vaccinations coincide with the age at which symptoms of ASD usually appear. This review supposes that the developmental derangement of ASD does not begin at this time, but instead is a continuation of maladaptive development that began early in the womb. Evidence has been presented indicating that the cortical disorganization of ASD represents prenatal dysfunction of cortical layer formation and differentiation (Bauman and Kemper, 2005; Stoner et al., 2014). While we are also asserting that aberrant microglial functioning and synaptic pruning continues postnatally, the initial insult must have occurred prenatally to derange the process of cortical layer formation and differentiation. Therefore, childhood vaccinations could not explain our proposed etiology of ASD. On the other hand, early gestational insults could impact the lifelong functioning of microglia, thereby affecting both prenatal cortical organization and postnatal synaptogenesis.

\section{Microglia}

Traditionally, microglia have been likened to macrophages of the brain and ascribed the same process of polarization. That is to say that research has operated on the assumption that microglia polarize in the same way as tissue macrophages. This canonical way of thinking outlines a resting state, an M1 state and an M2 state of microglia. The resting state of microglia is the baseline, where microglia reside before and after polarization and microglia in this state are chiefly responsible for the pruning of extraneous synapses during postnatal neural development (Paolicelli et al., 2011; Wake et al., 2009). The M1 or classical state is neurotoxic and activated in response to harmful stimuli. In contrast, the M2 or alternative state has been associated with transforming growth factor beta as well as brain derived neurotrophic factor and has proven to be involved in regeneration after an inflammatory response (Fig. 2) (Cherry et al., 2014; Gupta et al., 2014; Xia et al., 2015). Microglial activation involves resting microglia activating to the M1 state in response to an inflammatory event. In contributing to the inflammatory response, the M1 microglia damage the surrounding neural environment. The microglia then alter their polarization to the M2 state to repair the nearby tissues and promote growth (Cherry et al., 2014; Gupta et al., 2014).

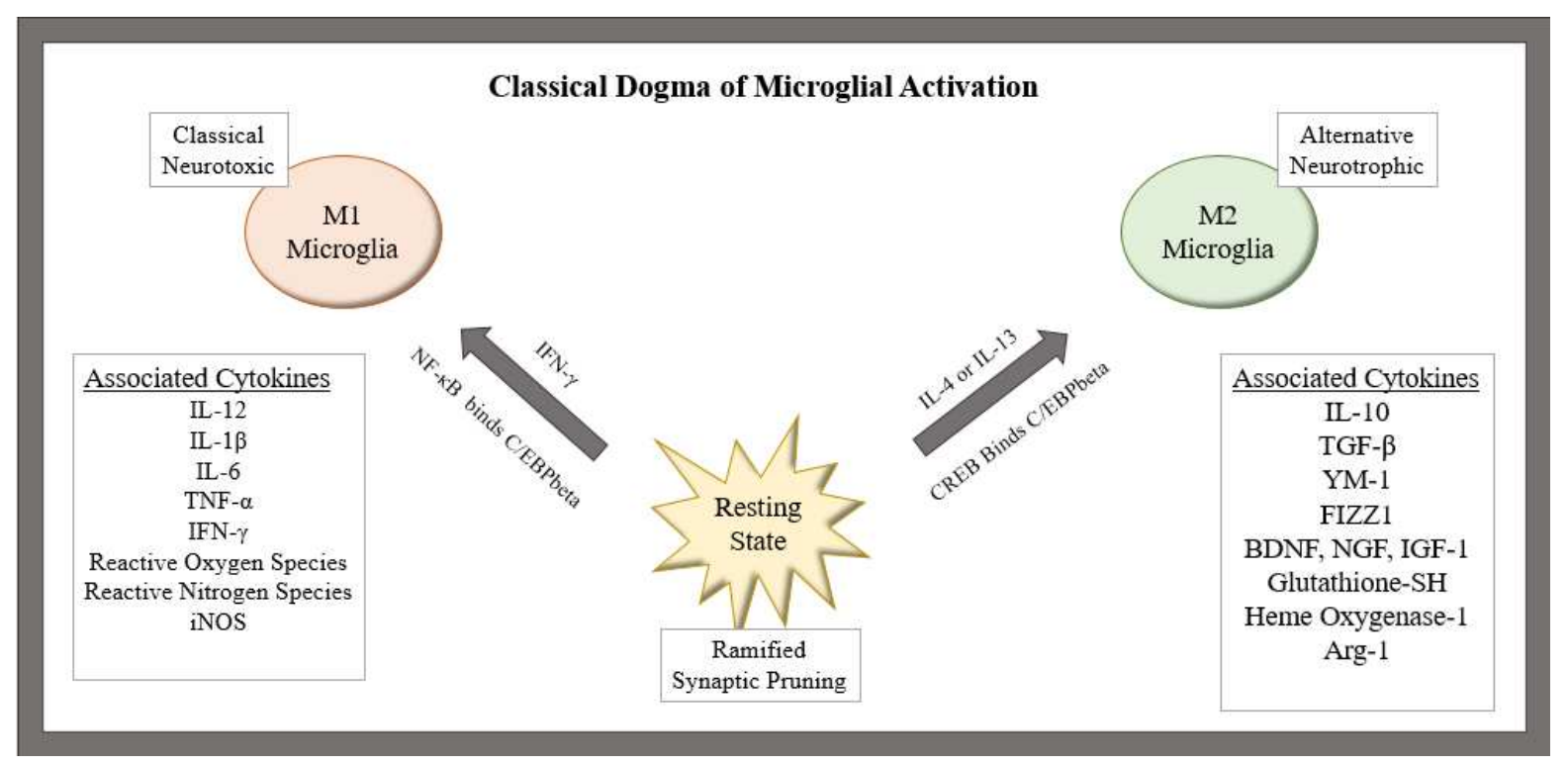

Fig. 2. Diagram outlining the activation patterns of microglia 
Classically activated, M1 microglia are a response to harmful stimuli. Specifically, interferon- $\gamma$ released by Th1 CD4+ T Cells, astroglia, or other microglia is responsible for triggering the M1 activation state (Cherry et al., 2014) Additionally, the transcription factor NF- $\kappa \mathrm{B}$ has been shown to activate the M1 subtype when it binds the C/EBPbeta gene (Gorgoni et al., 2002; Paolicelli et al., 2011; Xia et al., 2015). Once in the M1 state, the production of MHC II, CD86 and Fcyreceptors is up regulated and additional CD4 $+\mathrm{T}$ cells are activated (Paolicelli et al., 2011). These receptors aid the cell in antigen presentation and cell-cell cross talk. Furthermore, M1 cells are significant source of the cytokine IL-12 as well as reactive oxygen and nitrogen species (Paolicelli et al., 2011; Xia et al., 2015). Additional pro-inflammatory cytokines produced by classically activated microglia include IL-1 $\beta$, IL-6, TNF$\alpha$ and interferon gamma (Xia et al., 2015). A key enzyme that has been used in the identification of M1 activated microglia is inducible Nitric Oxide Synthase (iNOS) which generates nitric oxide out of arginine.

The alternatively activated, M2 subtype can be induced by IL-4 or IL-13 (Cherry et al., 2014; Chhor et al., 2013). In addition, it has been demonstrated that the transcription factor CREB interacts with $\mathrm{C} / \mathrm{EBPbeta}$ as well as CREB Binding Protein (CBP), to initiate the alternative subtype response (Ruffell et al., 2009). It is notable that $\mathrm{C} / \mathrm{EBPbeta}$ is therefore involved in the activation of both the M1 and M2 subtypes. CREB and $\mathrm{NF}-\kappa \mathrm{B}$ compete for binding with $\mathrm{C} / \mathrm{EBP}$ beta in order to activate their respective subtypes (Xia et al., 2015). Once activated, the alternative state produces the enzyme arginase 1 (Arg-1), which converts arginine to polyamines, proline and orthinines (Cherry et al., 2014). Arg-1 will effectively outcompete iNOS produced by the M1 subtype for the use of arginine (Xia et al., 2015). The M2 phase also produces a large amount of IL-10, which inhibits the production of the pro-inflammatory mediators IL-1 $\beta$, IL-6 and TNF- $\alpha$. IL-10 also up regulates Glutathione-SH (GSH) and Nerve Growth Factor (NGF) and both of these mediators inhibit apoptosis by suppressing caspase 3 (Cherry et al., 2014; Wake et al., 2009). This subtype also up regulates Heme Oxygenase-1 which functions in combination with Glutathione-SH as an antioxidant (Wake et al., 2009). Transforming growth factor beta is associated with the M2 state and is responsible for playing a role in neurogenesis as well as up regulating the anti-apoptotic proteins Bcl-2 and Bcl-x1 (Xia et al., 2015). Also involved in the M2 response are YM-1, IGF-1 and FIZZ1. These markers are involved with matrix deposition and tissue repair (Cherry et al., 2014; Stoner et al., 2014). Finally, Brain Derived Neurotrophic Factor (BDNF) is secreted by M2 cells (Gupta et al., 2014). BDNF and NGF function neurotrophically by increasing the production of new neurons and myelin sheaths (Gorgoni et al., 2002).To summarize, the effects of the M2 subtype of microglia are neurotrophic and will override the neurotoxic effects of the M1 subtype thereby leading to regenerative neural overgrowth.

New evidence has cast doubt on this classical dogma of microglial activation (Ransohoff, 2016; Xue et al., 2014). While it now does seem unlikely that microglia exist in 3 discrete states, ultimately the issue is a matter of semantics. Regardless of whether microglia polarize or vary their function along a spectrum, the fact remains that the neurotoxic and neurotrophic factors released by microglia are under precise control. Each pattern of mediator release can be induced separately via specific interleukins and micro RNAs. A loss of balance between the neurotoxic and neurotrophic mediators could be responsible for deficits in cortical organization and synaptic pruning.

While it has long been known that microglia are abnormally active in ASD patients, recently it was discovered that the active microglia in ASD patients exist primarily in an M2-like state (Gesundheit et al., 2013; Gupta et al., 2014). This review proposes that epigenetic dysregulation of microglial activation favorable to an M2-like state leads to cortical disorganization and dendritic overgrowth that is ultimately responsible for the appearance of the symptoms of ASD between 12 and 36 months of age. During this period in normal postnatal development, dendritic spine density peaks (Hutsler and Zhang, 2010). Following the peak, resting state microglia prune extraneous synapses and dendritic density is reduced to normal levels (Paolicelli et al., 2011; Wake et al., 2009). If the microglia were predisposed to an M2-like state during post-natal development, then the dendritic spines would tend to overgrow due to the elevated proportion of neurotrophic modulators and the tendency for the neurotrophic effects of microglia to override the neurotoxic ones. This dendritic overgrowth would be amplified by the inability of activated microglia to prune unnecessary synapses. The resultant cluttering of the brain would only begin to be evident between the ages of 12 and 36 months when dendritic density would exceed its natural peak. This proposed mechanism might also explain the increase in head circumference and brain volume observed during the early childhood of ASD patients (Sacco et al., 2015). An overabundance of neurotrophic factors released from M2-like microglia might also explain the prenatal cortical malformation observed in ASD patients. The formation and differentiation of cortical layers, could easily be deranged by inappropriate levels of growth factors released by aberrant microglia.

Epigenetic preconditioning, or priming, of microglia has also been demonstrated. Specifically, primary infections have been shown to increase the sensitivity of 
microglia and to persistently amplify the response to future events (Harry and Kraft, 2012). This altered pattern of activation in response to a secondary event has even been demonstrated in fetuses following a gestational insult (Cao et al., 2015). Aberrant preconditioning of microglia that favors the neurotrophic state could be responsible for alterations in the ability of microglia to orchestrate cortical differentiation and synaptogenesis.

\section{Potential Therapeutic Interventions}

Before potential interventions can be discussed, it must be mentioned that some key points of this proposed etiology still need to be scientifically demonstrated. In vitro bench work must be conducted to prove that there is indeed a causal association between neurotrophic, M2-like microglia, disordered cortical growth and dendritic overgrowth. Additionally, work should also be undertaken to show that those aberrant growth patterns are causally associated with the autistic neurobehavioral phenotype. If those causal associations can be properly established, then work could begin to exploit this pathway for therapeutic intervention.

With all that being said, this proposed etiology of ASD naturally leads to several potential targets for therapeutic intervention. Following this etiology, the primary goal of therapeutic intervention should be to either counteract the preconditioning of microglia, or to reduce the M2-like activation of microglia. By shifting the balance of microglia back towards the resting state, dendritic overgrowth could be pruned back and if the intervention was performed early enough, cortical disorganization might be avoided. In this way, these therapies hold the promise of ameliorating the symptoms of ASD.

These goals could be met, at least partially, by counseling expectant mothers. The risk of initiating microglial malfunction and thus developing ASD could be addressed very early on by discussing ways by which mothers could avoid potential gestational insults. Emphasis should be placed on maintaining optimum nutrition and minimizing stress throughout the pregnancy. This method would primarily function as prophylaxis to prevent the initial stimulus from occurring. Additionally, it is possible that eliminating stress and improving nutrition after an insult has occurred might have a positive effect on fetal microglia. These counseling sessions should be complimented with thorough medical histories. Special attention could be paid to women with a personal history of psychosocial stress or a family history of congenital neurobehavioral disorders.

A true intervention, that is a therapy applied after the disease is already underway, might involve targeting the micro RNAs associated with M2-like microglia. Specifically miRNA-145, miRNA-214 and miRNA-
124 are associated with a shift to an M2-like state and could be targeted for down regulation via the introduction of specifically tailored small inhibitory RNA's (Kaminska et al., 2016). As an example, inhibition of a micro RNA associated with the M1 state, miR-204, was able to reverse conditions induced by activation to an M1-like state (Li et al., 2015). While existing work has focused on mitigating the neurotoxic damage from M1-like microglia, similar methods could certainly be employed to reduce the overgrowth induced by overactive, M2-like microglia.

Another intervention might target the interaction between CREB and C/EBP-beta. Pharmaceutical interference at this point might prevent excessive activation to a harmful M2-like state. C/EBP-beta is a particularly interesting target because both the M1 and M2-like states are activated through it via $N F-\kappa \beta$ and CREB respectively. A therapy that blocks C/EBP-beta might indiscriminately block activation to both the M2 and M1-like states, thereby trapping microglia in the desirable resting state. As evidence of this, one study found that administration of miRNA-124 directly inhibits C/EBP-beta and transforms microglia into a resting-like state (Ponomarev et al., 2011). Any therapies that aim to directly alter the levels of microglial activation must keep the balance between damaging and regenerative effects in mind. Something that exclusively blocks the M2-like state could shift the balance to the neurotoxic, M1-like state and the consequences of that would be disastrous.

Perhaps the most difficult part of implementing any of these interventions would be identifying children with significantly high enough risk of ASD to warrant intervention. Progress has been made in this area by a group of researchers who demonstrated that by performing serial MRI scans on high risk patients, increases in cortical surface area growth could be used to accurately predict an ASD diagnosis (Hazlett et al., 2017). While these results are promising, they have not yet been repeated and serial MRI scans are likely to be prohibitively expensive. Additionally, this new MRI technique still only predicts ASD at 24 months and maximal benefit from these therapies would likely only be achieved with earlier implementation. This is due to the fact that at age 2 or later, the dendritic density has already peaked. Since synaptic remodeling is an ongoing, postnatal process, some improvement could probably be achieved even if therapy was introduced at this late age. Regardless, postnatal therapy would be unable to counteract the issues of cortical disorganization that occur early in the womb. Therefore, the ideal therapy would be applied in utero. Again, the issue of patient identification arises, but perhaps this kind of therapy could instead be applied prophylactically. If we were able to develop a 
pharmaceutical that could safely and reliably prevent the neurotrophic preconditioning of fetal microglia, it could be applied to high risk women.

\section{Conclusion}

Though the initial data is promising, research in this area is still sorely lacking. The pathogenesis we are proposing presents several opportunities for therapeutic intervention, but it is contingent on causal associations that haven't yet been demonstrated. The prospect of altering the course of Autism Spectrum Disorder is something that we cannot afford to miss and we hope that this paper will spur additional research into this topic.

\section{Acknowledgment}

Authors are thankful to the Neag Cancer Center at the University of Connecticut Health Center and to the Department of Medicine at the University of Connecticut School of Medicine for their full support of this article.

\section{Author's Contributions}

Ryan Slovak: Contributed to the literature search, planning of the paper, figure design, editing and revising.

Nitya G. Chakraborty: Contributed to the writing as well as theliterature search, planning, figure design, editing and revising.

\section{Ethics}

All authors have read and approved this manuscript. We confirm that the content is original and that there are no conflicts of interest to report.

\section{References}

Bauman, M.L. and T.L. Kemper, 2005. Neuroanatomic observations of the brain in autism: A review and future directions. Int. J. Dev. Neurosci., 23: 183187. DOI: 10.1016/j.ijdevneu.2004.09.006

Beversdorf, D.Q., S.E. Manning, A. Hillier, S.L. Anderson and R.E. Nordgren et al., 2005. Timing of prenatal stressors and autism. J. Autism. Dev. Disord., 35: 471-478. DOI: 10.1007/s10803-005-5037-8

Brod, S., L. Rattazzi, G. Piras and F. D'Acquisto, 2014. 'As above, so below' examining the interplay between emotion and the immune system. Immunology, 143: 311-318.

DOI: $10.1111 / \mathrm{imm} .12341$

Cao, M., M. Cortes, C.S. Moore, S.Y. Leong and L.D. Durosier et al., 2015. Fetal microglial phenotype in vitro carries memory of prior in vivo exposure to inflammation. Front Cell Neurosci., 9: 294-294. DOI: 10.3389/fncel.2015.00294
Cherry, J.D., J.A. Olschowka and M.K. O'Banion, 2014. Neuroinflammation and M2 microglia: The good, the bad and the inflamed. J. Neuroinflammat., 11: 98-98. DOI: 10.1186/1742-2094-11-98

Chhor, V., T. Le Charpentier, S. Lebon, M.V. Oré and I.L. Celador et al., 2013. Characterization of phenotype markers and neuronotoxic potential of polarised primary microglia in vitro. Brain Behav. Immunity, 32: 70-85.

DOI: $10.1016 /$ j.bbi.2013.02.005

Christensen, D.L., J. Baio, K. Van Naarden Braun, D. Bilder and J. Charles et al., 2016. Prevalence and characteristics of autism spectrum disorder among children aged 8 years--autism and developmental disabilities monitoring network, 11 sites, United States, 2012. MMWR Surveill. Summ., 65: 1-23. DOI: $10.15585 / \mathrm{mmwr} . \mathrm{ss} 6503 \mathrm{a} 1$

Connors, S.L., P. Levitt, S.G. Matthews, T.A. Slotkin and M.V. Johnston et al., 2008. Fetal mechanisms in neurodevelopmental disorders. Pediatr. Neurol., 38: 163-176. DOI: 10.1016/j.pediatrneurol.2007.10.009

Courchesne, E., K. Campbell and S. Solso, 2011. Brain growth across the life span in autism: age-specific changes in anatomical pathology. Brain Res., 1380: 138-145. DOI: 10.1016/j.brainres.2010.09.101

Courchesne, E., C.M. Karns, H.R. Davis, R. Ziccardi and R.A. Carper et al., 2001. Unusual brain growth patterns in early life in patients with autistic disorder: An MRI study. Neurology, 57: 245-254.

de La Rochebrochard, E. and H. Joshi, 2013. Children born after unplanned pregnancies and cognitive development at 3 years: Social differentials in the United Kingdom millennium cohort. Am. J. Epidemiol., 178: 910-920. DOI: 10.1093/aje/kwt063

Feldman, D., A. Banerjee and M. Sur, 2016. Developmental dynamics of rett syndrome. Neural Plast., 2016: 6154080-6154080.

DOI: $10.1155 / 2016 / 6154080$

Finer, L.B. and M.R. Zolna, 2011. Unintended pregnancy in the United States: Incidence and disparities, 2006. Contraception, 84: 478-485.

DOI: 10.1016/j.contraception.2011.07.013

Gardener, H., D. Spiegelman and S.L. Buka, 2009. Prenatal risk factors for autism: A comprehensive meta-analysis. Brit. J. Psychiatry, 195: 7-14. DOI: 10.1192/bjp.bp.108.051672

Gesundheit, B., J.P. Rosenzweig, D. Naor, B. Lerer and D.A. Zachor et al., 2013. Immunological and autoimmune considerations of Autism Spectrum Disorders. J. Autoimmun., 44: 1-7. DOI: $10.1016 /$ j.jaut.2013.05.005

Gorgoni, B., D. Maritano, P. Marthyn, M. Righi and V. Poli, 2002. C/EBP beta gene inactivation causes both impaired and enhanced gene expression and inverse regulation of IL-12 p40 and p35 mRNAs in macrophages. J. Immunol., 168: 4055-4062. 
Gupta, S., S.E. Ellis, F.N. Ashar, A. Moes and J.S. Bader et al., 2014. Transcriptome analysis reveals dysregulation of innate immune response genes and neuronal activity-dependent genes in autism. Nat. Commun., 5: 5748-5748.

DOI: $10.1038 /$ ncomms6748

Harris, J.C., 2016. The origin and natural history of autism spectrum disorders. Nat. Neurosci., 19: 1390-1391. DOI: 10.1038/nn.4427

Harry, G.J. and A.D. Kraft, 2012. Microglia in the developing brain: A potential target with lifetime effects. Neurotoxicology, 33: 191-206.

DOI: 10.1016/j.neuro.2012.01.012

Hazlett, H.C., H. Gu, B.C. Munsell, S.H. Kim and M. Styner et al., 2017. Early brain development in infants at high risk for autism spectrum disorder. Nature, 542: 348-351. DOI: 10.1038/nature21369

Hutsler, J.J. and H. Zhang, 2010. Increased dendritic spine densities on cortical projection neurons in autism spectrum disorders. Brain Res., 1309: 83-94. DOI: 10.1016/j.brainres.2009.09.120

Kaminska, B., M. Mota and M. Pizzi, 2016. Signal transduction and epigenetic mechanisms in the control of microglia activation during neuroinflammation. Biochim. Biophys. Acta, 1862: 339-351. DOI: 10.1016/j.bbadis.2015.10.026

Lee, S.Y., A.R. Lee, R. Hwangbo, J. Han and M. Hong et al., 2015. Is oxytocin application for autism spectrum disorder evidence-based? Exp. Neurobiol., 24: 312-324.

DOI: $10.5607 /$ en.2015.24.4.312

Leigh, J.P. and J. Du, 2015. Brief report: Forecasting the economic burden of autism in 2015 and 2025 in the United States. J. Autism Dev. Disord., 45: 41354139. DOI: $10.1007 / \mathrm{s} 10803-015-2521-7$

Li, L., Q. Sun, Y. Li, Y. Yang and Y. Yang et al., 2015. Overexpression of SIRT1 Induced by Resveratrol and Inhibitor of miR-204 suppresses activation and proliferation of microglia. J. Mol. Neurosci., 56: 858-867. DOI: $10.1007 / \mathrm{s} 12031-015-0526-5$

Ma, Q., F. Xiong and L. Zhang, 2014. Gestational hypoxia and epigenetic programming of brain development disorders. Drug Discovery Today, 19: 1883-1896. DOI: 10.1016/j.drudis.2014.09.010

Matthews, S.G., 2002. Early programming of the hypothalamo-pituitary-adrenal axis. Trends Endocrinol. Metab., 13: 373-380.

Morgan, J.T., G. Chana, C.A. Pardo, C. Achim and K. Semendeferi et al., 2010. Microglial activation and increased microglial density observed in the dorsolateral prefrontal cortex in autism. Biol. Psychiatry, 68: 368-376.

DOI: $10.1016 /$ j.biopsych.2010.05.024
Nardone, S. and E. Elliott, 2016. The interaction between the immune system and epigenetics in the etiology of autism spectrum disorders. Front Neurosci., 10: 329. DOI: 10.3389/fnins.2016.00329

Paolicelli, R.C., G. Bolasco, F. Pagani, L. Maggi and M. Scianni et al., 2011. Synaptic pruning by microglia is necessary for normal brain development. Science, 333: 1456-1458.

DOI: $10.1126 /$ science. 1202529

Park, H.R., J.M. Lee, H.E. Moon, D.S. Lee and B.N. Kim et al., 2016. A short review on the current understanding of autism spectrum disorders. Exp. Neurobiol., 25: 1-13. DOI: 10.5607/en.2016.25.1.1

Ponomarev, E.D., T. Veremeyko, N. Barteneva, A.M. Krichevsky and H.L. Weiner, 2011. MicroRNA-124 promotes microglia quiescence and suppresses EAE by deactivating macrophages via the C/EBP-alpha-PU.1 pathway. Nat. Med., 17: 64-70. DOI: $10.1038 / \mathrm{nm} .2266$

Ransohoff, R.M., 2016. A polarizing question: Do M1 and M2 microglia exist? Nat. Neurosci., 19: 987-991. DOI: $10.1038 / \mathrm{nn} .4338$

Ruffell, D., F. Mourkioti, A. Gambardella, P. Kirstetter and R.G. Lopez et al., 2009. A CREB-C/EBPbeta cascade induces M2 macrophage-specific gene expression and promotes muscle injury repair. Proc. Natl. Acad. Sci. USA, 106: 17475-17480.

DOI: 10.1073/pnas.0908641106

Sacco, R., S. Gabriele and A.M. Persico, 2015. Head circumference and brain size in autism spectrum disorder: A systematic review and meta-analysis. Psychiatry Res., 234: 239-251.

DOI: 10.1016/j.pscychresns.2015.08.016

Squarzoni, P., M.S. Thion and S. Garel, 2015. Neuronal and microglial regulators of cortical wiring: Usual and novel guideposts. Front Neurosci., 9: 248.

DOI: $10.3389 /$ fnins. 2015.00248

Stiles, J. and T.L. Jernigan, 2010. The basics of brain development. Neuropsychol. Rev., 20: 327-348. DOI: $10.1007 / \mathrm{s} 11065-010-9148-4$

Stoner, R., M.L. Chow, M.P. Boyle, S.M. Sunkin and P.R. Mouton et al., 2014. Patches of disorganization in the neocortex of children with autism. New Engl. J. Med., 370: 1209-1219. DOI: 10.1056/NEJMoa1307491

Talge, N.M., C. Neal and V. Glover, 2007. Antenatal maternal stress and long-term effects on child neurodevelopment: How and why? J. Child Psychol. Psychiatry, 48: 245-261. DOI: $10.1111 /$ j.1469-7610.2006.01714.x

Taylor, L.E., A.L. Swerdfeger and G.D. Eslick, 2014. Vaccines are not associated with autism: An evidence-based meta-analysis of case-control and cohort studies. Vaccine, 32: 3623-3629. DOI: $10.1016 /$ j.vaccine.2014.04.085 
Van den Bergh, B.R., E.J. Mulder, M. Mennes and V. Glover, 2005. Antenatal maternal anxiety and stress and the neurobehavioural development of the fetus and child: links and possible mechanisms. A review. Neurosci. Biobehav. Rev., 29: 237-258. DOI: 10.1016/j.neubiorev.2004.10.007

Wake, H., A.J. Moorhouse, S. Jinno, S. Kohsaka and J. Nabekura, 2009. Resting microglia directly monitor the functional state of synapses in vivo and determine the fate of ischemic terminals. J. Neurosci., 29: 3974-3980.

DOI: 10.1523/jneurosci.4363-08.2009

Wakefield, A.J., S.H. Murch, A. Anthony, J. Linnell and D.M. Casson et al., 1998. Ileal-lymphoid-nodular hyperplasia, non-specific colitis and pervasive developmental disorder in children. Lancet, 351: 637-641.

Ward, A.J., 1990. A comparison and analysis of the presence of family problems during pregnancy of mothers of "autistic" children and mothers of normal children. Child Psychiatry Hum. Dev., 20: 279-288.

Xia, C.Y., S. Zhang, Y. Gao, Z.Z. Wang and N.H. Chen, 2015. Selective modulation of microglia polarization to M2 phenotype for stroke treatment. Int. Immunopharmacol., 25: 377-382.

DOI: 10.1016/j.intimp.2015.02.019
Xue, J., S.V. Schmidt, J. Sander, A. Draffehn and W. Krebs et al., 2014. Transcriptome-based network analysis reveals a spectrum model of human macrophage activation. Immunity, 40: 274-288. DOI: 10.1016/j.immuni.2014.01.006
Abbreviations
ASD - Autism Spectrum Disorder, Th1- Type 1 Helper $\mathrm{T}$ Cells, NF- $\kappa \mathrm{B}$ - Nuclear Factor kappa light chain enhancer of activated B cells, C/EBPbeta - CCAAT (Cytosine-Cytosine-Adenosine-Adenosine-
Thymidine)/Enhancer Binding Protein beta, MHC II - Major Histocompatibility Complex II, IL - interleukin, TNF- $\alpha$ - Tumor necrosis factor alpha, iNOS - inducible Nitric Oxide Synthase, CREB - cAMP (cyclic Adenosine Monophosphate) Response Element Binding Protein, Arg-1 - Arginase-1, GSH - Glutathione-SH (sulfhydryl group), NGF - Nerve Growth Factor, Bcl - $\mathrm{B}$ cell lymphoma, Bcl-xl - B cell lymphoma extra large, IGF-1 - Insulin Like Growth Factor, BDNF - Brain Derived Neurotrophic Factor, miRNA - Micro Ribonucleic Acid 